\title{
Instruction Compression in Runtime for Embedded Systems
}

\author{
Wanderson Roger Azevedo Dias, Raimundo da Silva Barreto \\ Universidade Federal do Amazonas - Departamento de Ciência da Computação \\ Manaus, Amazonas, Brasil \\ \{roger, rbarreto\}@dcc.ufam.edu.br \\ Edward David Moreno \\ Universidade Federal de Sergipe - Departamento de Ciência da Computação \\ Aracaju, Sergipe, Brasil \\ edwdavid@gmail.com
}

\begin{abstract}
The efficient use of embedded systems relies heavily on appropriate strategies to optimize the execution time and power consumption. These systems are characterized by resource restrictions, including the amount of memory available for applications. However, there are several techniques that make the embedded systems more efficient. One of those techniques is the code compression; the proposals found in the analyzed literature assume that the code is compressed at compilation time and decompressed at runtime. This article proposes the development of a new method of compression and decompression (on-the-fly) called of MIC (Middle Instruction Compression). The MIC was compared with the Huffman method and both were implemented in hardware using VHDL and FPGA. The results of our experiments showed that the MIC achieved better performance when compared to Huffman for some programs from MiBench. We have reduced $17 \%$ the number of logical elements of FPGA and $6 \%$ clock frequency (in $\mathrm{MHz}$ ) and $42 \%$ rate of compression.
\end{abstract}

\section{Introdução}

Sistemas embarcados são quaisquer sistemas digitais que estejam inseridos a outros sistemas com a finalidade de acrescentar ou otimizar funcionalidades [14]. Os sistemas embarcados têm por função monitorar e/ou controlar o ambiente no qual esteja inserido. Esses ambientes podem estar presentes em dispositivos eletrônicos, eletrodomésticos, veículos, máquinas, motores e muitas outras aplicações.

A crescente demanda pelo uso de sistemas embarcados tem se tornado cada vez mais comum, motivando a implementação de complexos sistemas em um único chip, os chamados System-on-Chip (SoC). Neste caso, o processador embarcado é um dos principais componentes dos sistemas computacionais embarcados [3]. Atualmente, muitos processadores embarcados encontrados no mercado são baseados em arquiteturas de alto-desempenho (por exemplo, as arquiteturas RISC de 32 bits) que garantem um melhor desempenho computacional para as tarefas a serem executadas. Consequentemente, o projeto de sistemas embarcados para processadores de alto desempenho não é uma tarefa simples.

Sabe-se que muitos dos sistemas embarcados são alimentados por baterias. Por essa razão, é de suma importância que estes sistemas sejam capazes de controlar e gerenciar sua potência, possibilitando assim a redução no consumo de energia e no controle do aquecimento. Portanto, projetistas e pesquisadores concentraram-se no desenvolvimento de técnicas que diminuam o consumo de energia mantendo os requisitos de desempenho. Uma dessas técnicas é a compressão do código das instruções em memória.

A grande maioria das técnicas, metodologias e padrões de desenvolvimento de software, para o controle e o gerenciamento do consumo de energia, não se mostra viável para o desenvolvimento de sistemas embarcados, devido aos mesmos possuírem inúmeras limitações de recursos computacionais e físicos [10]. As atuais estratégias concebidas para o controle e o gerenciamento no consumo de energia, foram desenvolvidas para sistemas de propósitos gerais, em que os custos adicionais de processadores ou memórias são geralmente insignificantes [14].

O tamanho de código aumenta significativamente na medida em que os sistemas tornam-se mais heterogêneos e complexos. Neste sentido, surgiu uma técnica de alto nível que procura comprimir o código em tempo de compilação e a respectiva descompressão, por sua vez, é feita em tempo de execução $[10,11,12]$. 
A técnica de compressão foi desenvolvida com o intuito de reduzir o tamanho de um código [13]. Mas no decorrer do tempo, grupos de pesquisadores verificaram que essa técnica poderia trazer grandes benefícios para o desempenho e o consumo de energia nos sistemas de propósitos gerais e nos sistemas embarcados. A partir do momento que o código em memória está comprimido é possível em cada requisição do processador buscar uma quantidade bem maior de instruções contidas na memória. Assim, haverá uma diminuição nas atividades de transição nos pinos de acesso à memória, levando a um possível aumento no desempenho do sistema e a uma possível redução no consumo de energia do circuito [13].

Da mesma forma, quando se armazenam instruções comprimidas na memória cache, aumenta-se o número de instruções armazenadas na cache e aumenta-se sua taxa de acertos (hit rate), diminuindo a busca na memória principal, aumentando o desempenho do sistema e, por conseguinte, diminuindo o consumo de energia.

Este artigo apresenta o desenvolvimento de um novo método de compressão/descompressão de instruções (em tempo de execução), que foi implementado em VHDL (Very High Speed Integrated Circuit Hardware Description Languages) e prototipado em uma FPGA (Field Programmable Gate Array), denominado de MIC (Middle Instruction Compression), o qual foi comparado com o tradicional método de Huffman também implementado em hardware, e mostrou ter mais eficiência computacional que o método de Huffman a partir de uma comparação usando o benchmark MiBench [5].

O restante do artigo está organizado da seguinte forma: a Seção 2 apresenta os trabalhos correlatos; a Seção 3 explana a arquitetura PDCCM desenvolvida para o método $M I C$; a Seção 4 detalha a compressão em tempo de execução; descreve o método $M I C$ e a implementação realizada em FPGA e por fim, a Seção 6 apresenta as conclusões e idéias para trabalhos futuros.

\section{Trabalhos Correlatos}

Nesta seção são mostradas algumas arquiteturas para a execução de códigos de instruções comprimidas, encontradas na literatura.

WOLFE \& CHANIN [15] desenvolveram o CCRP (Compressed Code RISC Processor), que foi o primeiro hardware descompressor implementado em um processador RISC (MIPS R2000) e também foi a primeira técnica a usar as falhas de acesso à cache para acionar o mecanismo de descompressão.

O CCRP tem uma arquitetura idêntica ao padrão do processador RISC e assim os modelos dos programas são inalterados. Isso implica em que todas as ferramentas de desenvolvimento existentes para a arquitetura RISC, incluindo compiladores otimizados, simuladores funcionais, bibliotecas gráficas e outras, também servem para a arquitetura CCRP.

A unidade de compressão usada é a linha da cache de instruções. A cada falha de acesso à cache, as instruções são buscadas na memória principal, descomprimidas e alimentam a linha da cache onde houve a falha [15]. O fato de o CCRP já fazer a descompressão das instruções antes de armazená-las na cache é vantajoso, no sentido que os endereços de saltos contidos na cache são os mesmos do código original. Isto resolve a maioria dos problemas de endereçamento, não havendo necessidade de recorrer a artifícios como: (I) Colocar hardware extra no processador para tratamento diferenciado dos saltos; e (II) Fazer patches de endereços de salto.

A técnica CCRP utilizou o algoritmo de Huffman [6] gerado através de um histograma de ocorrências de bytes de programa e mostrou uma razão de compressão de $73 \%$, em média, para o pacote testado (composto pelos programas: nasal, nasa7, tomcatv, matrix $25 A$, espresso, fpppp e outros). Para modelos de memórias mais lentos DRAM (Dynamic Random Access Memory), o desempenho do processador foi na maioria das vezes suavemente melhorado. Para modelos mais rápidos de memória EPROM (Erasable Programmable Read Only Memory), o desempenho sofreu uma leve degradação.

AZEVEDO [2] propôs um método chamado de IBC (Instruction Based Compression), que tem por função realizar a divisão do conjunto de instruções do processador em classes, levando em consideração a quantidade de ocorrências juntamente com número de elementos de cada classe. Pesquisas realizadas por AZEVEDO [2] mostraram melhores resultados na compressão de 4 classes de instruções. O método de compressão desenvolvido consiste em agrupar pares no formato [prefixo, codeword] que substituem o código original. Nos pares formados, o prefixo indica a classe da instrução e o codeword serve como um índice para a tabela de instruções.

O processo de descompressão é realizado em 4 estágios de pipeline. O primeiro estágio é chamado de INPUT onde é convertido o endereço do processador (código não comprimido) em endereço da memória principal. O segundo estágio é chamado de FETCH, que é responsável pela busca da palavra comprimida na memória principal. O terceiro estágio é conhecido como $D E C O D E$ onde verdadeiramente é realizada a decodificação dos codewords. E finalmente no quarto estágio, chamado de OUTPUT, é realizada a consulta no dicionário de instrução para ser fornecida a instrução ao processador. Nos testes realizados, AZEVEDO [2] obteve uma taxa de compressão de $53,6 \%$ para o processador MIPS (Microprocessor without Interlocked Pipeline Stages) e 61,4\% para o processador SPARC (Scalable Processor Architecture). Quanto ao 
desempenho, constatou-se uma perda de 5,89\% utilizando o método IBC.

BENINI et al [3] desenvolveram um algoritmo de compressão que é adaptado para execução eficiente do hardware (descompressor). As instruções são compactadas em grupos que têm o tamanho de uma linha da cache e a sua descompactação ocorre no instante que são extraídas da cache. Experimentos foram realizados com o processador DLX, devido o mesmo ter uma arquitetura simples de 32 bits e também ser uma arquitetura RISC. Além disso, o processador DLX é semelhante a vários processadores comerciais da família ARM (Advanced RISC Machine) [1] e MIPS. Uma tabela de 256 posições foi utilizada para guardar as instruções mais executadas. Cada linha da cache é formada por 4 instruções originais ou um conjunto de instruções comprimidas e possivelmente intercaladas com outras não comprimidas, prefixado por uma palavra de 32 bits. A palavra não comprimida tem um posicionamento fixo na linha da cache e serve para diferenciar uma linha de cache com instruções comprimidas das outras linhas com as instruções originais. De fato, uma linha de cache comprimida não contém necessariamente todas as instruções comprimidas, mas sempre deve ter um número entre 5 e 12 instruções comprimidas na linha da cache para ser vantajoso o uso da compressão [3].

Para evitar o uso das tabelas de tradução de endereços, BENINI et al, exigem que os endereços de destino estejam sempre alinhados a 32 bits (palavra). A primeira palavra ( 32 bits) da linha de cache contém uma marca L e um conjunto de bits de flags. A marca é um opcode de instrução não utilizada, ou seja, um opcode inválido que sinaliza uma linha comprimida (no processador DLX os opcodes são de 6 bits).

O algoritmo de compressão desenvolvido por BENINI et al [3], analisa o código sequencialmente, a partir da primeira instrução (supondo que cada linha da cache já esteja alinhada) e tenta acondicionar instruções adjacentes em linhas comprimidas. Os experimentos realizados em vários pacotes de código $\mathrm{C}$ do benchmark fornecido pelo projeto Ptolemy [4], comprovaram que houve uma redução média de $28 \%$ no tamanho do código e de $30 \%$ no consumo de energia.

LEKATSAS et al [8, 9], desenvolveram uma unidade de descompressão com um único ciclo. A descompressão pode ser aplicada para instruções de qualquer tamanho de um processador RISC (16, $24 \mathrm{ou}$ 32 bits). A única aplicação específica é a parte do interfaceamento entre o processador e a memória (principal ou cache). O mecanismo de descompressão é capaz de descomprimir uma ou duas instruções por ciclo para atender a demanda da CPU sem aumentar o tempo de execução. Foi desenvolvida uma técnica para criar um dicionário que contém as instruções que aparecem com mais frequência. $\mathrm{O}$ dicionário de código refere-se a uma classe de métodos de compressão que substitui sequencias de símbolos com os índices de uma tabela. Essa tabela é chamada de "dicionário" e os índices são os "codewords" no programa compactado [9]. A principal vantagem dessa técnica é que os índices geralmente são de comprimento fixo, e assim, simplifica a lógica da descompressão em acessar o dicionário e também reduz a latência da descompressão.

Os resultados obtidos nos testes realizados demonstraram que houve um ganho médio de $25 \%$ de desempenho no tempo de execução dos aplicativos usando a compressão de código e uma média de $35 \%$ na redução do tamanho do código. Essa tecnologia desenvolvida não está limitada em apenas um processador, mas sim pode ser aplicada e obter resultados similares em outros processadores.

LEFURGY et al [7], propuseram uma técnica de compressão de código baseado na codificação do programa usando um dicionário de códigos. Assim, a compressão é realizada após a compilação do código fonte, porém, o código objeto é analisado e as sequencias comuns de instruções são substituídas por uma palavra codificada (codeword), como na compressão de texto. Apenas as instruções mais frequentes são comprimidas. Um bit (escape bit) é utilizado para distinguir uma palavra comprimida (codificada) de uma instrução não comprimida. As instruções correspondentes às instruções comprimidas são armazenadas em um dicionário no hardware de descompressão. As instruções comprimidas são usadas para indexar as entradas do dicionário. O código final consiste de codewords misturadas com instruções não comprimidas.

Observa-se que um dos problemas mais comuns encontrados na compressão de código se refere à determinação dos endereços alvo das instruções de salto. Normalmente este tipo de instrução (desvio direto) não é codificado para evitar a necessidade de reescrever as palavras de códigos que representam estas instruções [6]. Já os desvios indiretos podem ser codificados normalmente, pois, como seus endereços alvos estão armazenados em registradores, apenas as palavras de códigos necessitam ser rescritas. Neste caso, é necessária apenas uma tabela para mapear os endereços originais armazenados no registrador para os novos endereços comprimidos.

Este método diverge dos demais métodos vistos na literatura no sentido que, os endereços alvos estejam sempre alinhados a 4 bits (tamanho de um codeword) e não ao tamanho da palavra do processador (32 bits). Como vantagem destaca-se uma melhor compressão; mas como desvantagem verifica-se a necessidade de alterações no core do processador (um hardware extra) para tratar desvios para endereços alinhados a 4 bits. Entretanto, não fica claro os detalhes sobre a interação do hardware descompressor com os processadores 
experimentados (PowerPC, ARM e i386). O funcionamento do hardware descompressor é realizado basicamente da seguinte maneira: A instrução é buscada da memória, caso seja um codeword, a lógica de decodificação dos codewords obtém o deslocamento e o tamanho do codeword que servirá como índice para acessar a instrução não comprimida no dicionário e repassar ao processador. No caso de instruções não comprimidas, elas são repassadas diretamente ao processador. Com o método proposto em [7], foram obtidas taxas de compressões de $61 \%$ para o processador PowerPC, 66\% para o processador ARM e $75 \%$ para o processador i386. As métricas de desempenho e consumo de energia não foram expressas.

\section{Arquiteturas para Compressão}

$\mathrm{Na}$ literatura são encontrados dois tipos básicos de arquiteturas de compressão de código, CDM e PDC, que indicam o posicionamento do descompressor em relação ao processador e subsistema de memória, como mostra a Figura 1. Segundo NETTO [10], a arquitetura CDM (Cache Decompressor Memory) indica que o descompressor está posicionado entre a cache e a memória principal, enquanto que a arquitetura PDC (Processor Decompressor Cache) posiciona o descompressor entre o processador e a cache.

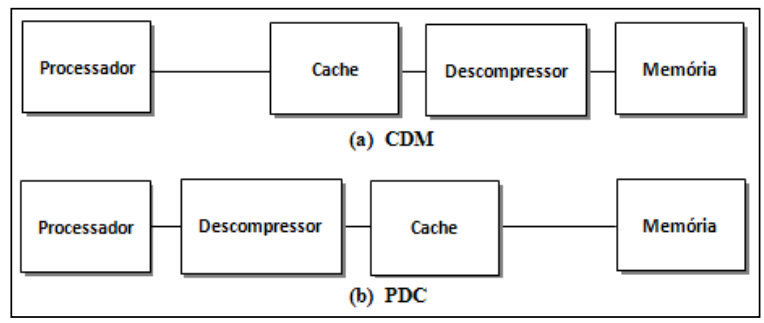

Figura 1. Arquiteturas de descompressão de código: (a) CDM e (b) PDC [10]

Como foi visto anteriormente (Seção 2), o desenvolvimento de arquiteturas para compressão ou descompressão de código de instrução é feito de forma separada, ou seja, na grande maioria dos trabalhos desenvolvidos só é tratado o hardware descompressor porque a compressão das instruções geralmente é feita por meio de modificações no compilador. Assim, a compressão é realizada em tempo de compilação e a descompressão é feita em tempo de execução usando um hardware específico para descompressão.

Para o funcionamento do método $M I C$, proposto neste trabalho, foi necessário o desenvolvimento de uma nova arquitetura, em hardware, que realize tanto a compressão quanto a descompressão dos códigos de instruções em tempo de execução. A arquitetura criada foi intitulada de PDCCM (Processor Decompressor
Cache Compressor Memory) no qual é mostrado que o hardware de compressão foi inserido entre as memórias cache e principal e o hardware de descompressão foi inserido entre o processador e a memória cache. A arquitetura PDCCM foi implementada em VHDL e prototipada em uma FPGA Cyclone-II modelo EP2C20F484C7 do fabricante ALTERA ${ }^{\circledR}$.

A arquitetura PDCCM trabalha com instruções do tamanho de 32 bits, ou seja, cada linha da cache de instrução é composta por 4 bytes. Assim, a arquitetura desenvolvida é compatível com sistemas que usam o processador ARM como núcleo do seu sistema embarcado, pois este processador tem como característica um conjunto de instruções de 32 bits. $\mathrm{Na}$ arquitetura PDCCM, usando o método de compressão/descompressão $M I C$ todas as instruções que serão salvas na cache de instrução sofrerão uma compressão de $50 \%$ no seu tamanho original.

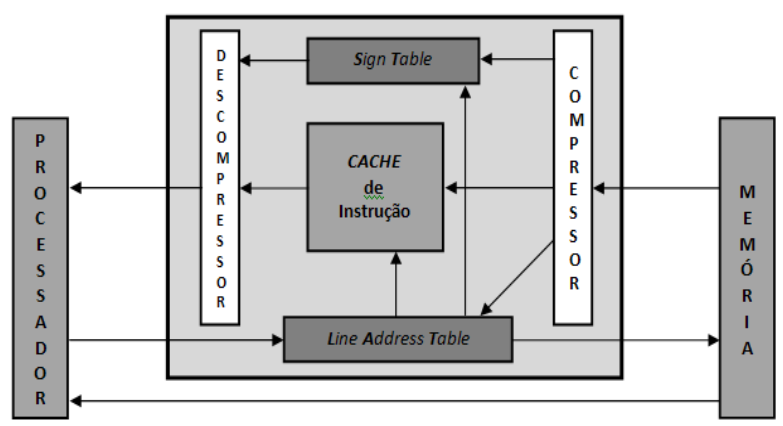

Figura 2. Arquitetura PDCCM

A Figura 2 mostra a arquitetura PDCCM desenvolvida para implantar o novo método de compressão/descompressão de instruções em hardware $(M I C)$, que é composta por quatro componentes básicos, sendo eles:

- LAT (Tabela da Linha de Endereços - Line Address Table): é uma tabela que tem por função fazer o mapeamento dos endereços das instruções com o seu novo endereço na cache de instrução;

- ST (Tabela de Sinais - Sign Table): é uma tabela que contém bits que servem como flags para indicar ao descompressor qual dupla de bits deverá ser reconstituída, ou seja, descomprimida;

- Compressor: tem por função fazer a compressão de todos os códigos das instruções que serão salvas na cache de instrução. O compressor é acionado toda vez que houver um miss na cache de instrução;

- Descompressor: tem por função fazer a descompressão de todas as instruções que estão armazenadas na cache de instrução e serão repassadas ao processador. O descompressor é 
acionado toda vez que houver um hit na cache de instrução.

\section{Compressão em Tempo de Execução}

Assim como a tabela de Huffman usada por WOLFE e CHANIN [15] na arquitetura do CCPR; a tabela de instrução e tabela de tradução de endereço usada por AZEVEDO [2] na arquitetura do IBC; o dicionário de instruções usada por BENINI et al [3] e o dicionário de códigos usado por LEKATSAS et al [8, 9] e LEFURGY et al [7], o método MIC também requer tabelas adicionais que serão usadas pelos componentes ST e LAT, para armazenarem o conjunto de flags da instrução comprimida e o mapeamento dos novos endereços das instruções comprimidas na cache, respectivamente.

Portanto, mesmo com o acréscimo adicional das tabelas (ST e LAT) no método $M I C$, que inicialmente são grandes ao ponto de parecer não ter tido ganho na compressão pois se requer uma tabela adicional equivalente à metade do tamanho da cache existente, $\mathrm{e}$ sendo a compressão de $50 \%$, não há ganho no uso de memória, considerado como recurso físico. Apesar disso, essas tabelas foram inseridas para avaliarmos a estrutura da nova arquitetura e do método proposto, que levanta a opção de ter compressão em tempo de execução, sendo diferente das outras propostas que baseiam-se na construção de dicionários em tempo de compilação. Neste momento o grupo está trabalhando com o $M I C$ no intuito de retirar (ou diminuir) essas tabelas adicionais e assim obter resultados mais expressivos num futuro próximo.

Para compressão, a cada instrução que for lida na memória RAM e salva na cache de instrução será dividida em duplas de bits, sendo cada dupla formada por: 00, 01, 10 e 11. O compressor $M I C$ realiza a seguinte lógica: duplas com bits iguais (00 ou 11) são substituídas pelo bit 0 (zero) e duplas com bits diferentes (10 ou 01) são substituídas pelo bit 1 (um). Assim, uma dupla de bits é reduzida a um bit único.

Uma tabela auxiliar (ST) é usado para guardar o conjunto de flags da dupla de bits comprimidos. Em duplas de bits que iniciam com o valor 0 (zero), como no caso 00 ou 10, é salvo na ST o bit 0 e em duplas de bits que iniciam com o valor 1 (um), como no caso 11 ou 01 , é salvo na ST o bit 1 . Vale ressaltar que o modo de endereçamento das linhas de instrução para essa arquitetura é Big-Endian.

Portanto, o método MIC (Middle Instruction Compression) é um método de compressão que tem por função reduzir em $50 \%$ o tamanho dos códigos de instruções que são salvas na cache de instrução, passando então o tamanho dessas instruções de 32 bits (tamanho original) para 16 bits (tamanho comprimido).
Para um melhor esclarecimento e sempre que possível, os nomes dos componentes, variáveis e pinos de entrada e saída (input e output) são semelhantes aos usados no código implementado em VHDL.

\subsection{Descrição do Método $M I C$}

$\mathrm{O}$ processador requisita uma instrução à cache através de um pino, que para essa implementação foi chamado de end_inst_proc (PC atual). Será pesquisada na LAT a existência ou não do endereço fornecido pelo processador. Se a instrução for encontrada na cache a LAT sinalizará com um ACERTO (hits). Então, a LAT fornecerá o novo endereço da instrução na cache de instrução, o endereço do conjunto de flags da instrução na ST e o posicionamento da dupla de bytes (primeira ou segunda), ou seja, onde se encontra a instrução e os flags na cache de instrução e na ST, respectivamente. Todas essas informações são repassadas ao descompressor que descomprimirá a instrução e a retornará de forma descomprimida ao processador através da variável returnd_inst_proc.

A descompressão dos códigos de instrução é realizada da seguinte forma:

- O novo endereço da instrução que foi repassado pela LAT, foi localizado na cache de instrução e na ST;

- A cache de instrução e a ST retornam para o descompressor os 16 bits da instrução comprimida e os 16 bits do conjunto de flags;

- Se o bit lido da instrução comprimida na cache de instrução for 0 (zero), a dupla de bits a ser reconstituída será 00 ou 11 . O que definirá qual será a dupla de bits é o bit flag, ou seja, se o bit flag for 0 a dupla de bits a ser reconstituída será 00 e se o bit flag for 1 a dupla de bits a ser reconstituída será 11 ;

- Mas se o bit lido da instrução comprimida na cache de instrução for 1 (um), a dupla de bits a ser reconstituída será 10 ou 01 . Então, novamente o bit flag que definirá qual será a dupla de bits, ou seja, se o bit flag for 0 a dupla de bits a ser reconstituída será 10 e se o bit flag for 1 a dupla de bits a ser reconstituída será 01 ;

- Para cada instrução a ser descomprimida, são analisados os 16 bits da instrução salva na cache de instrução, transformando assim as instruções comprimidas de 16 bits em instruções descomprimidas de 32 bits.

Agora, se o endereço fornecido pelo processador não se encontrar na LAT, significa que não existe essa instrução na cache de instrução. A LAT sinalizará uma FALHA (miss) na linha da cache de instrução. O endereço fornecido pelo processador será repassado 
para a memória RAM (Random Access Memory), onde a mesma será consultada e verificada se existe ou não essa instrução. Caso a pesquisa na RAM indique uma FALHA, a instrução será buscada em uma memória auxiliar flash. Agora se a pesquisa indicar um ACERTO significa que a instrução encontra-se na RAM. Em seguida, a RAM retornará uma cópia da instrução no formato original (não comprimida) para o processador através da variável returnc_inst_proc e outra cópia para o compressor, que realizará todo o processo de compressão.

A compressão dos códigos de instrução é realizada da seguinte forma:

- A instrução é localizada na memória RAM, uma cópia da mesma é repassada para o processador e outra para o compressor;

- A instrução no compressor é dividida em 16 duplas de bits, sendo que cada dupla é formada no instante que é lida pela função de compressão. $\mathrm{O}$ início da leitura da instrução vinda da memória RAM é pelo modo MSB (bit mais significativo);

- O compressor sempre analisará em qual parte da dupla de bytes (primeira ou segunda) deverá ser salva a instrução comprimida na cache de instrução e na ST;

- Se a dupla de bits lida para a compressão for $00 \mathrm{ou}$ 11, então essa dupla de bits será substituída pelo bit 0 e salva na cache de instrução. Agora se a dupla de bits lida for 10 ou 01 então essa dupla de bits será substituída pelo bit 1 e salva na cache de instrução;

- O conjunto de flags da ST será formado através da seguinte lógica: se o primeiro bit da dupla de bits que está sendo comprimido for 0 , então o bit flag salvo será 0 . Agora se o primeiro bit da dupla de bits que está sendo comprimido for 1, então o bit flag salvo será 1;

- Após o compressor fazer toda a compressão dos 32 bits da instrução original nos 16 bits comprimidos e seus respectivos bits flags, o compressor irá salvar na dupla de bytes (primeira ou segunda) a instrução comprimida na cache de instrução e o conjunto de flags na ST;

- A tabela LAT será atualizada com o novo endereço da instrução salva na cache de instrução;

- Para cada instrução que for buscada na memória RAM, repetirá esse processo de compressão.

Importante salientar que essa técnica de compressão/descompressão é realizada em tempo de execução, via hardware específico que foi prototipado em FPGA. Na implementação em hardware verificou-se que é similar aos trabalhos de LEKATSAS [8, 9], obtivemos um componente que precisa de apenas um único ciclo para o processo de compressão ou descompressão, além dos benefícios mostrados na próxima seção.

Para uma análise de desempenho do método desenvolvido neste projeto (MIC), foi implementado em hardware o tradicional método de compressão de Huffman, pois o mesmo foi utilizado por WOLFE \& CHANIN [15] na arquitetura do CCRP e também por BENINI et al [3] e LEFURGY et al [7]. Portanto, a comparação do MIC com Huffman permite verificar pontos fortes e fracos desta nova abordagem em compressão de códigos em relação a um método já conceituado e altamente usado no meio cientifico.

\subsection{Implementação em FPGAs}

Os benchmark utilizados nas simulações de compressão e descompressão dos métodos $M I C$ e Huffman são do pacote MiBench [5] específicos para sistemas embarcados e de categorias diferentes, os quais estão em código Assembly do processador ARM9, conforme obtido através da ferramenta IDA Pro [17]. A categoria e funcionalidade dos benchmark MiBench usados nas simulações são:

- Dijsktra (Rede de Computadores): é um algoritmo que calcula o menor caminho em um grafo;

- FFT (Telecomunicação): é um algoritmo que executa a Transformada de Fourier usado no tratamento de sinais digitais para encontrar as frequências em um sinal de entrada;

- MAD (Dispositivos de Consumo): é um decodificador de áudio MPEG de alta qualidade;

- QuickSort (Controle Automotivo e Industrial): é um algoritmo que faz ordenação de dados;

- SHA (Segurança): é um algoritmo que gera chaves de criptografia para troca segura de dados e assinaturas digitais;

- Stringsearch (Automação): é um algoritmo que faz a busca de uma string em um texto.

Foi utilizado o conjunto de instruções do processador embarcado ARM (família ARM9, versão ARM922T, ISA ARMv4T) para simular o funcionamento do compressor e descompressor dos métodos MIC e Huffman na arquitetura PDCCM. No entanto, esse processador escolhido (ARM) é do tipo RISC e possui um conjunto de instruções formado por 32 bits (instrução) que o habilitou como sendo uma boa plataforma para simular a arquitetura PDCCM.

Vale ressaltar que a única alteração necessária na arquitetura PDCCM para o uso do método de Huffman foi a troca do componente da ST pela a HT (Tabela de Huffman) que contem a árvore de Huffman dos códigos das instruções comprimidas.

Para as simulações de compressão/descompressão dos métodos MIC e Huffman, foram selecionadas as 4.096 primeiras instruções de cada MiBench (devido as 
limitações físicas do FPGA usado para a prototipação), obtidas através do código compilado (Assembly) para o processador embarcado ARM, formando assim o conjunto de sequências de instruções que foram usadas para carregar um trecho das memórias RAM e a cache de instrução. Para mais detalhes, ver em [16].

O trecho da memória RAM descrita em VHDL foi utilizada em todas as simulações com os benchmark MiBench e teve tamanho fixo de 4.096 linhas de 4 bytes cada, contabilizando assim 131.072 bits e a cache de instrução teve o tamanho de 512 linhas de 32 bits cada. Assim, se observa que há uma relação de 8:1 entre os tamanhos da memória RAM e da respectiva cache de instruções. A Tabela 1 mostra as métricas nas estatísticas de desempenho e na Tabela 2 é possível visualizar as métricas na temporização da arquitetura PDCCM no FPGA; ambas usando os métodos MIC e Huffman na compressão/descompressão das instruções de alguns programas do MiBench.

Tabela 1. Estatística de desempenho do FPGA

\begin{tabular}{lcc}
\hline & MIC & Huffman \\
\hline & Compressão \\
\hline Elementos Lógicos & $1.460(32 \%)$ & $1.317(28 \%)$ \\
Registradores & $825(18 \%)$ & $738(16 \%)$ \\
Pinos & $177(56 \%)$ & $170(54 \%)$ \\
\hline \multicolumn{3}{c}{ Descompressão } \\
\hline Elementos Lógicos & $3.464(74 \%)$ & $4.414(94 \%)$ \\
Registradores & $1.045(23 \%)$ & $1.482(32 \%)$ \\
Pinos & $177(56 \%)$ & $170(54 \%)$ \\
\hline
\end{tabular}

Observando a Tabela 1, no processo de compressão, o método de Huffman mostrou ser um pouco mais eficiente no uso dos recursos do FPGA para a arquitetura PDCCM, sendo que a quantidade de elementos lógicos, registradores e pinos entre os dois métodos não passou de $4 \%$ de diferença a favor do método de Huffman. Já no processo de descompressão, o método MIC apresentou melhores resultados nos recursos do FPGA, sendo que a quantidade de elementos lógicos, registradores e pinos ficaram em média aproximadamente $9 \%$ a menos a favor do método MIC.

Tabela 2. Temporização do FPGA

\begin{tabular}{lcc}
\hline & MIC & Huffman \\
\hline & Compressão & \\
\hline Tempo no pior caso & $9.228 \mathrm{~ns}$ & $9.712 \mathrm{~ns}$ \\
Clock em MHz & $75.98 \mathrm{MHz}$ & $69.88 \mathrm{MHz}$ \\
Clock em tempo & $13.257 \mathrm{~ns}$ & $14.579 \mathrm{~ns}$ \\
\hline & Descompressão & \\
\hline Tempo no pior caso & $8.632 \mathrm{~ns}$ & $10.708 \mathrm{~ns}$ \\
Clock em MHz & $68.42 \mathrm{MHz}$ & $66.97 \mathrm{MHz}$ \\
Clock em tempo & $14.668 \mathrm{~ns}$ & $14.900 \mathrm{~ns}$ \\
\hline
\end{tabular}

Nota-se na Tabela 2, que o método MIC apresentou melhor temporização no FPGA para todos os benchmark MiBench analisados. $\mathrm{Na}$ compressão, é observada uma diferença de $6.1 \mathrm{MHz}$ (na frequência do clock em $\mathrm{MHz}$ ) a mais para o método MIC sendo este um dos pontos que o torna mais eficiente que o método de Huffman. Já o tempo, no pior caso, para os dois métodos foram bem idênticos. Na descompressão, são observados que o clock em $\mathrm{MHz}$ e em tempo são idênticos para os dois métodos, sendo que o método $M I C$ tem uma pequena vantagem comparada ao método de Huffman.

Tendo como base as primeiras $4 \mathrm{~K}$ instruções dos programas, obtidas através do código Assembly compilado para a plataforma ARM, observamos na Tabela 3 que o método MIC comprimiu em 50\% o tamanho das instruções, ou seja, após o processo de compressão o programa passou de $4 \mathrm{~K}$ para apenas $2 \mathrm{~K}$ linhas na cache de instrução. Enquanto isso o método de Huffman obteve uma média geral na compressão de $30 \%$ a menos em relação ao tamanho da memória RAM usada na simulação.

Tabela 3. Comparativo na taxa de compressão

\begin{tabular}{lcc}
\hline \multicolumn{1}{c}{ MiBench } & MIC & Huffman \\
\hline Dijsktra & $2.048(50 \%)$ & $2.622(36 \%)$ \\
FFT & $2.048(50 \%)$ & $2.803(32 \%)$ \\
MAD & $2.048(50 \%)$ & $3.002(27 \%)$ \\
QuickSort & $2.048(50 \%)$ & $3.245(21 \%)$ \\
SHA & $2.048(50 \%)$ & $2.785(32 \%)$ \\
StringSearch & $2.048(50 \%)$ & $2.913(29 \%)$ \\
\hline \multicolumn{1}{c}{ Médias } & $\mathbf{2 . 0 4 8 ( 5 0 \% )}$ & $\mathbf{2 . 8 9 5 ( 3 0 \% )}$ \\
\hline
\end{tabular}

Com base nos resultados apresentados, constatamos que para a arquitetura PDCCM usando as 4.096 primeiras instruções dos benchmarks MiBench (Dijsktra, FFT, MAD, QuickSort, SHA e StringSearch), o método MIC mostrou-se mais eficiente na taxa de compressão, ou seja, um percentual de $\approx 42 \%$ menor (para o tamanho do código final) se comparado ao método de Huffman para todos os MiBenchs analisados.

\section{Conclusões e Trabalhos Futuros}

Este artigo descreveu um novo método de compressão, chamado de $M I C$, o qual foi prototipado em FPGAs, e mostrou-se viável para os sistemas embarcados que usam arquitetura RISC. Pois futuramente esta técnica pode tornar-se um componente necessário em projetos de sistemas embarcados. Com a utilização das técnicas de compressão de código, as arquiteturas RISC conseguem minimizar um de seus maiores problemas, que é a quantidade de memória para armazenar os programas.

Mediante as simulações realizadas com alguns programas do benchmark MiBench verificamos que o 
método MIC apresentou as seguintes médias: 17\% a menos na utilização dos elementos lógicos do FPGA, uma frequência $(\mathrm{MHz})$ de operação em aproximadamente $6 \%$ maior para os processos de compressão/descompressão dos códigos de instruções e $42 \%$ mais eficiente na taxa de compressão dos MiBench analisados em relação ao método de Huffman, que também foi prototipado em hardware.

Portanto, analisando os dados obtidos por meio das simulações, apesar da memória adicional usada pelo dicionário (ST) do método $M I C$, conclui-se que o método desenvolvido e apresentado neste artigo mostrou ser mais eficiente computacionalmente em comparação com o método de Huffman também implementado em hardware. As simulações utilizaram aplicações de categorias diferentes, sendo eles: Dijsktra, FFT, MAD, QuickSort, SHA e StringSearch do benchmark MiBench para obter as medições de desempenho.

Como trabalhos futuros ficam: realizar medições reais do consumo de energia da arquitetura PDCCM usando os métodos MIC e Huffman; projetar e implementar um processador de arquitetura RISC que já tenha o hardware compressor e descompressor embutido em seu núcleo; realizar testes de compressão e descompressão usando os métodos MIC e Huffman com mais programas do benchmark MiBench e chegar a uma implementação em ASIC, de modo que este projeto transcenda o âmbito acadêmico, servindo como uma contribuição, também, para o meio industrial.

\section{Referências}

[1] ARM. An Introduction to Thumb. Advanced RISC Machines (ARM) Ltd., March 1995.

[2] R. J. de Azevedo. Uma Arquitetura para Código Comprimido em Sistemas Dedicados. Tese de Doutorado, Instituto de Computação da UNICAMP, Brasil, Junho de 2002. 136 p.

[3] L. Benini, A. Macii and A. Nannarelli. "Cached-Code Compression for Energy Minimization in Embedded Processor". In Proceedings of the International Symposium on Low-Power, Electronics and Design (ISPLED'01). Huntington Beach, California, USA, August 2001, pp. 322-327.

[4] J. Davis II, M. Goel, C. Hylands, B. Kienhuis, E. A. Lee, J. Liu, X. Liu, L. Muliadi, S. Neuendorffer, J. Reekie, N. Smyth, J. Tsay and Y. Xiong. Overview of the Ptolemy Project, ERL Technical Memorandum UCB/ERL, Technical Report No M-99/37, Department of Electrical Engineering and Computer Science, Univ. of California, Berkeley, California, USA, July 6 , 1999.

[5] M. Guthaus, J. Ringenberg, D. Ernst, T. Austin, T. Mudge and R. Brown. "MiBench: A Free, Commercially Representative Embedded Benchmark
Suite". In Proceedings of the IEEE $4^{\text {th }}$ Annual Workshop on Workload Characterization (WWC-4). Austin, Texas, USA, December 2001, pp. 3-14.

[6] D. A. Huffman. "A Method for the Construction of Minimum-Redundancy Codes". Proceedings of the Institute of Radio Engineers (IRE), 40(9):1098-1101, September 1952.

[7] C. Lefurgy, P. Bird, I-C. Chen and T. Mudge. "Improving Code Density Using Compression Techniques". In Proceedings of $30^{\text {th }}$ Annual International Symposium on Microarchitecture (MICRO 30). Research Triangle Park, NC, USA, December 1997, pp. 194-203.

[8] H. Lekatsas, J. Henkel and V. Jakkula. "Design of One-Cycle Decompression Hardware for Performance Increase in Embedded Systems". In Proceedings of the $39^{\text {th }}$ Annual Design Automation Conference (DAC'02). New Orleans, Louisiana, USA, June 2002, pp. 34-39.

[9] H. Lekatsas and W. Wolf. "Code Compression for Embedded Systems". In Proceedings of the $35^{\text {th }}$ Annual Design Automation Conference (DAC'98). San Francisco, California, USA, June 1998, pp. 516-521.

[10] E. B. W. Netto. Compressão de Código Baseada em Multi-Profile. Tese de Doutorado, Instituto de Computação, Universidade Estadual de Campinas, Maio de 2004. 137 p.

[11] E. B. W. Netto, R. Azevedo, P. Centoducatte and G. Araújo. "Mixed Static/Dynamic Profiling for Dictionary Based Code Compression". In Proc. of the Intl. Symposium on System-on-Chip (SoC'03). Tampere, Finland, November 2003, pp. 159-163.

[12] E. B. W. Netto, R. Azevedo, P. Centoducatte and G. Araújo. "Multi-Profile Based Code Compression". In Proceedings of the $41^{\text {th }}$ Annual Design Automation Conference (DAC'04). San Diego, California, USA, June 2004, pp. 244-249.

[13] E. B. W. Netto, R. S. de Oliveira, R. Azevedo, P. Centoducatte. Compressão de Código em Sistemas Embarcados. HOLOS CEFET-RN. Ano 19, páginas 23-28, Dezembro, 2003. 94p.

[14] A. S. de Oliveira, F. S. de Andrade. Sistemas Embarcados - Hardware e Firmware na Prática. Editora Érica, 2006, 316p.

[15] A. Wolfe and A. Chanin. "Executing Compressed Programs on an Embedded RISC Architecture". In Proceedings of $25^{\text {th }}$ Annual Intl. Symposium on Microarchitecture (MICRO 25). Portland, Oregon, USA, December 1992, pp. 81-91.

[16] W. R. A. Dias. Arquitetura PDCCM em Hardware para Compressão/Descompressão de Instruções em Sistemas Embarcados. Dissertação de Mestrado, Departamento de Ciência da Computação da UFAM, Brasil Abril de 2009. 152 p.

[17] IDA - The Interactive Disassembler. Disponível em: http://www.hex-rays.com. Acessado em 03 de maio de 2010. 